Apidologie, 1982, 13 (4), 353 à 366.

\title{
ÉTUDE DE LA DISPERSION DU POLLEN PAR LE VENT ET DE L'IMPORTANCE DE LA POLLINISATION ANEMOPHILE CHEZ LE COLZA (BRASSICA NAPUS L., VAR. OLEIFERA METZGER)
}

\author{
J. MESQUIDA* et M. RENARD**
}

\begin{abstract}
RÉSUMÉ
Les auteurs ont étudié la dispersion par le vent des grains de pollen de colza (variété " Primor ") et ont vérifié leur efficacité pollinisatrice sur des lignées mâle-stériles sous cages.

Ils ont constaté que les grains de pollen de colza pouvaient être transportés par le vent jusqu'à $32 \mathrm{~m}$ mais que les $75 \%$ du pollen total ont été capturés à moins de $6 \mathrm{~m}$. Les grains de pollen transportés sur de grandes distances n'ont pas eu une grande incidence sur la mise à graines du colza. A plus de $24 \mathrm{~m}$ la pollinisation anémophile n'a assuré que les 4 à $16 \%$ des taux de nouaison et 1 à $4 \%$ de la production grainière. Mais à moins de $6 \mathrm{~m}$ ce sont 23 à $29 \%$ des taux de nouaison et 3 à $12 \%$ de la production grainière qui ont été imputables à la pollinisation anémophile sous-cages.
\end{abstract}

\section{INTRODUCTION}

La fleur de colza dispose de toutes les caractéristiques morphologiques, anatomiques et physiologiques d'une fleur entomophile. Le colza est d'ailleurs une plante mellifère très activement visitée par les abeilles domestiques en raison de l'abondance de son nectar et de la richesse de son pollen. Chaque fleur de colza peut fournir environ $1 \mathrm{mg}$ de pollen d'un jaune très vif. Les dimensions du grain sont : $\mathrm{P}=24 \mu$ et $\mathrm{E}=26 \mu\left(^{*}\right)$ d'après MAURizio et Louveaux (1965). Le pollen de colza est gluant, les grains s'agglomèrent en amas et adhèrent facilement à la pilosité très fournie du corps et des pattes

* I.N.R.A., laboratoire de recherches de la chaire de zoologie, domaine de La Motte-au-Vicomte, B.P. 29, 35650 Le Rheu.

** I.N.R.A., station d'amélioration des plantes, domaine de La Motte-au-Vicomte, B.P. 29, 35650 Le Rheu.

(*) $\mathbf{P}=$ diamètre selon l'axe polaire du grain. $\mathrm{E}=$ diamètre selon l'axe équatorial du grain. 
des insectes pollinisateurs. C'est un pollen d'une valeur nutritive élevée pour l'abeille. Il contient environ 4,9\% d'azote total (Louveaux, 1958).

Ainsi, par ses nombreuses et fréquentes visites, l'abeille domestique transportant de grandes quantités de pollen de colza, favorise aussi bien l'autopollinisation que la pollinisation croisée. Cependant le transport du pollen de colza peut aussi être assuré par le vent qui, d'après OLsson (1955), semble jouer un rôle important dans la pollinisation de cette plante.

Le vent agit de deux manières différentes, soit par chute du pollen en secouant les plantes et surtout les ramifications, favorisant ainsi, selon Williams (1978), l'autopollinisation, soit par simple transport du pollen d'une plante à une autre. Pour Olsson (1955), le pollen de colza peut être transporté par le vent jusqu'à environ $40 \mathrm{~m}$ de distance. En fait, celle-ci peut varier selon les cultivars (Olsson et Persson, 1958).

Etudiant plus particulièrement la dispersion par le vent des pollens anémophiles, de nombreux auteurs (Bateman, 1947; Griffiths, 1950; Proctor et Yeo, 1973; WANG et al., 1960; WRIGHT, 1953) ont noté que les plus importantes captures se font surtout près de la source d'émission du pollen, qu'elles diminuent rapidement ensuite avec la distance, mais qu'elles persistent jusqu'à des distances relativement importantes. Proctor et Yeo ont ajouté que cette dispersion du pollen se fait selon une distribution normale du type leptocurtique (1).

Dans nos études faites à Rennes (Ille-et-Vilaine) sur la pollinisation du colza mâlestérile en vue de la production de semences hybrides F 1 (MESQuida et RENARD, 1978, 1981), nous avons été amenés à nous intéresser à la dispersion du pollen de colza par le vent dans le but de définir la part de la pollinisation anémophile dans les dispositifs d'alternance de colzas fertiles et stériles.

Dans cette note, nous présentons les résultats des observations faites pendant deux années consécutives. L'efficacité pollinisatrice du pollen transporté par le vent à différentes distances de la source émettrice de pollen a été vérifiée sur les plantes mâlestériles protégées des insectes pollinisateurs au moyen de cages.

\section{MATERIEL ET MÉTHODES}

\section{Matériel végétal et dispositifs}

On a utilisé deux types de colza : un colza mâle-fertile (lignée " Primor ") et un colza mâle-stérile (stérilité mâle cytoplasmique stable d'origine radis, lignée $n^{\circ}$ 3-22-3-7, MEsquida et RENARD, 1981).

Deux essais ont été effectués, l'un en 1978 (essai I) et l'autre en 1979 (essai II).

Chaque essai a été effectué dans une parcelle de colza de $60 \times 30 \mathrm{~m}$ comprenant, dans le sens de sa plus petite dimension, deux bandes centrales de colza mâle-fertile de $6 \mathrm{~m}$ de large en tout, bordées de chaque côté, sur $35 \mathrm{~m}$ de large, de 10 bandes de colza mâle-stérile. Chaque bande était constituée de 8 li-

(1) Par opposition à une courbe normale dite platycurtique (DAGNELIE, 1969). 
gnes avec un espacement de $0,30 \mathrm{~m}$ entre les lignes et de $0,70 \mathrm{~m}$ entre les bandes. Les bandes étaient orientées d'E en $W$ pour l'essai $I$, de sorte que les 10 bandes mâle-stériles se trouvaient, les unes au $N$ et les autres au $\mathrm{S}$ des bandes fertiles émettrices de pollen. Dans le second essai effectué l'année suivante dans un autre lieu, les bandes étaient orientées du NNW au SSE et les 10 bandes mâle-stériles se trouvaient, les unes à l'ENE et les autres à l'WSW des bandes fertiles centrales.

\section{Captures de pollen}

a) Dispositifs

Les captures de pollen transporté par le vent ont été effectuées dans les bandes de colza mâle-stériles situées de chaque côté des bandes mâle-fertiles et à différentes distances de celles-ci :

$-3 \mathrm{~m} ; 6 \mathrm{~m} ; 12 \mathrm{~m}$ et $24 \mathrm{~m}$ pour l'essai $\mathrm{I}$;

$-1 \mathrm{~m} ; 6 \mathrm{~m} ; 15 \mathrm{~m}$ et $32 \mathrm{~m}$ pour l'essai II.

A chaque niveau de capture on a installé deux capteurs de pollen, l'un en plein champ et l'autre sous cages, soit au total 16 capteurs par essai.

On a utilisé des cages de $3 \times 3 \times 2 \mathrm{~m}$ recouvertes d'un tissu synthétique monofilament à ouverture de mailles de $2 \mathrm{~mm}$, interdisant tout accès vers l'intérieur des insectes pollinisateurs. Chaque cage recouvre un secteur de bande de colza mâle-stérile de $9 \mathrm{~m}^{2}$ de surface. On a observé avec ces cages une diminution de la vitesse du vent de $90 \%$ pour un vent de $2750 \mathrm{~m} / \mathrm{h}$ et de $60 \%$ pour un vent de $4500 \mathrm{~m} / \mathrm{h}$.

\section{b) Description des capteurs de pollen}

On a utilisé un type de capteur de pollen fixe (Fig. 1) à lame de verre verticale abritée, mis au point par Mesquida (non publié). Il s'inspire de celui qui fait appel à la force du vent de Hyde et WiLliams (1945). Il est constitué d'un tube métallique de $2 \mathrm{~m}$ de long, solidement fixé au sol, et d'un support de lames en position verticale ( 2 et 3 , Fig. 1), surmonté d'une plaque horizontale qui protège la lame de la pluie et du soleil (1, Fig. 1).

Le support de lames coulisse verticalement le long du tube, ce qui permet de le régler et de le fixer à tout moment à $10 \mathrm{~cm}$ environ au-dessus du plateau de floraison du colza (4, Fig. 1). Une lame de verre de $76 \times 26 \mathrm{~mm}$ est maintenue verticalement au support et fait face à la source d'émission de pollen (3, Fig. 1). La lame de verre est enduite d'une fine couche de glycérine pure selon la méthode préconisée par HYDE (1959). La glycérine a pour effet de fixer les grains de pollen transportés par le vent.

Un anémomètre enregistreur était installé en permanence dans chaque essai.

\section{Observations effectuées}

a) Captures de pollen

Les captures de pollen se font durant 24 heures. On a déterminé et dénombré au microscope les grains de pollen isolés ou agglomérés de chaque prise sous cage et en extérieur, sur une surface de $150 \mathrm{~mm}^{2}$ de lame. Dans un but de simplification, les nombres de grains ainsi obtenus ont été ramenés à l'unité de surface $\left(\mathrm{cm}^{2}\right)$. On a évité de faire des dénombrements des captures les jours de pluie.

\section{b) Efficacité pollinisatrice}

La pollinisation des plantes mâle-stériles, due au pollen transporté par le vent sous cage, a été comparée à celle due au vent et aux insectes de l'extérieur.

L'efficacité pollinisatrice a été vérifiée sur les caractères suivants :

$$
\text { taux de nouaisc }\left(\frac{\text { nombre de siliques }}{\text { nombre de fleurs totales }} \times 100\right) \text {, }
$$

composantes du rendement (nombre de graines par silique, poids de 1000 grains et production de graines de 1000 fleurs) de la ramification primaire de 10 plantes mâle-stériles tirées au hasard à chaque niveau de captures dans les cages et en extérieur. Les rendements en graines concernent la totalité des plantes récoltées sur une surface de $9 \mathrm{~m}^{2}$ aussi bien sous cages qu'en plein champ. 

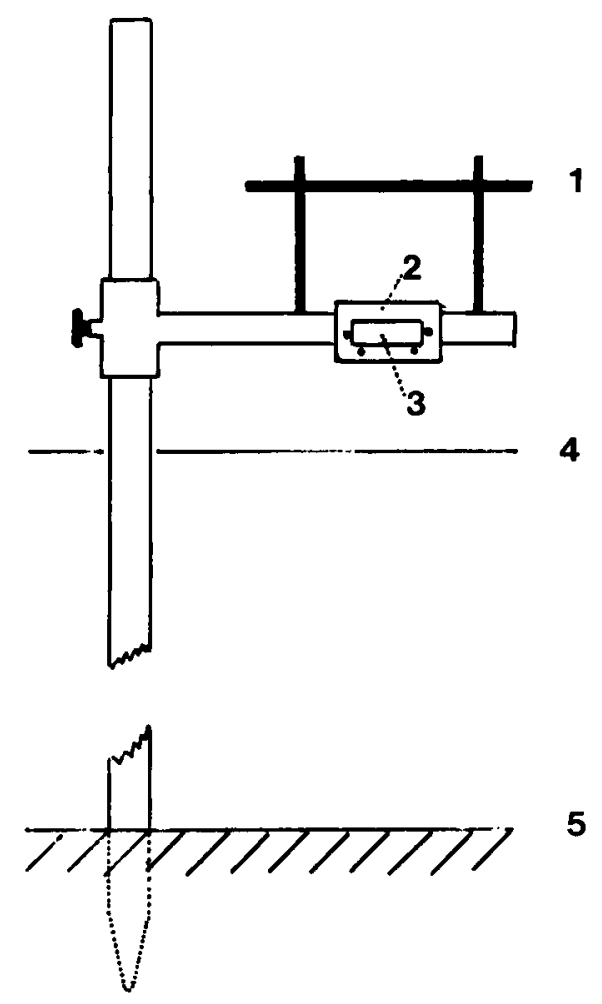

Fig. 1. - Schéma du capteur de pollen utilisé (d'après MESQUiDA).

1. - Protection métallique contre la pluie.

2. - Support de lames de verre.

3. - Lame de verre.

4. - Plateau de floraison de colza.

5. - Sol.

Échelle : 1/10.

FIG. 1. - Plan of the pollen collector.

1. - Metal protection against rain.

2. - Stand for glass slides.

3. - Glass slide.

4. - Platform for the flowering rapeseed.

5. - Ground.

Scale $: 1 / 10$.

\section{RÉSULTATS}

Les résultats obtenus permettent de faire les remarques suivantes :

\section{Distribution des captures}

Les captures des grains de pollen de colza transportés par le vent (tableau 1), en extérieur et sous cages, se sont effectuées jusqu'à $32 \mathrm{~m}$ de la bande mâle-fertile émettri- 
ce de pollen. Les plus grosses captures se sont produites à moins de $3 \mathrm{~m}$ (entre 42 et $51 \%$ du pollen total recueilli), elles ont été encore importantes à $6 \mathrm{~m}(24-39 \%$ du pollen total), elles ont diminué rapidement et graduellement ensuite : 10 à $21 \%$ entre $12 \mathrm{~m}$ et $15 \mathrm{~m}$; et 7 à $10 \%$ entre $24 \mathrm{~m}$ et $32 \mathrm{~m}$. C'est dans un secteur réduit, moins de $6 \mathrm{~m}$, que l'on a capturé la majeure partie du pollen transporté par le vent, soit $75 \%$ du pollen total.

TABL. 1. - Nombre moyen de grains de pollen de colza capturés par périodes de 24 heures et par $\mathrm{cm}^{2}$ dans les essais $I$ et II $\dot{a}$ différentes distances des plantes mâle-fertiles émettrices de pollen.

TABL. 1. - Mean number of rapeseed pollen grains per $\mathrm{cm}^{2}$ in 24-hour periods and in tests $I$ and $I I$ at various distances

from the male fertile plants producing pollen.

\begin{tabular}{|c|c|c|c|c|c|c|}
\hline \multicolumn{2}{|r|}{$\begin{array}{c}\text { Distance des captures } \\
\text { Distance of catchings }\end{array}$} & $3 \mathrm{~m}$ & $6 \mathrm{~m}$ & $12 \mathrm{~m}$ & $24 \mathrm{~m}$ & $x^{2}(1)$ \\
\hline \multirow{3}{*}{ I } & $\begin{array}{l}\text { Plein champ } \\
\text { In the open }\end{array}$ & $33( \pm 12)$ & $20( \pm 8)$ & $16( \pm 8)$ & $7( \pm 2)$ & \multirow{2}{*}{ NS } \\
\hline & $\begin{array}{l}\text { Sous cages } \\
\text { In cages }\end{array}$ & $13( \pm 5)$ & $12( \pm 5)$ & $3( \pm 1)$ & $3( \pm 1)$ & \\
\hline & $\begin{array}{c}(b) /(a) \\
\text { en } \%\end{array}$ & 39 & 60 & 19 & 43 & \\
\hline & $\begin{array}{c}\text { Distance des captures } \\
\text { Distance of catchings }\end{array}$ & $1 \mathrm{~m}$ & $6 \mathrm{~m}$ & $15 \mathrm{~m}$ & $32 \mathrm{~m}$ & \\
\hline \multirow{3}{*}{ II } & $\begin{array}{l}\text { Plein champ } \\
\text { In the open }\end{array}$ & $50( \pm 15)$ & $24( \pm 13)$ & $18( \pm 10)$ & $7( \pm 2)$ & \multirow{2}{*}{ NS } \\
\hline & $\begin{array}{l}\text { Sous cages } \\
\text { In cages }\end{array}$ & $30( \pm 15)$ & $14( \pm 7)$ & $10( \pm 5)$ & $5( \pm 3)$ & \\
\hline & $\begin{array}{l}(b) /(a) \\
\text { en } \%\end{array}$ & 60 & 58 & 56 & 71 & \\
\hline
\end{tabular}

(1) Hypothèse : en fonction des différentes distances les distributions des captures sous cages ne sont pas diffẻrentes de celles de plein champ.

$\mathrm{NS}=\chi^{2}$ non significatif $=$ hypothèse acceptée.

* $=\chi^{2}$ significatif à $\mathrm{P}=5 \%=$ hypothèse rejetée.

** $=\chi^{2}$ significatif à $\mathrm{P}=1 \%=$ hypothèse rejetée.

*** $=\chi^{2}$ significatif à $\mathrm{P}=1 \% \mathrm{o}=$ hypothèse rejetée.

$( \pm): \pm$ erreur type : $\pm s / \sqrt{ } n(s=$ écart-type $)$.

(1) Hypothesis : the distance of dispersal of pollen grains in cages are not different from those in the open over the various distances.

NS $=\chi^{2}$ not significant $=$ hypothesis corroborated

* $x^{2}$ significant at $\mathrm{P}=5 \%=$ hypothesis rejected.

$* *=X^{2}$ significant at $\mathrm{P}=1 \%=$ hypothesis rejected.

${ }^{* * *}=\chi^{2}$ significant at $\mathbf{P}=1 \% 0=$ hypothesis rejected. 
TABL. 2. - Comparaison de la pollinisation des colzas mâle-stériles

en plein champ et sous cages de l'essai I à différentes distances des plantes mâle-fertiles émettrices de pollen.

TABL. 2. - Comparison of the pollination of male sterile rapeseeds in the open and in cages in test $I$ at various distances from the male fertile plants producing pollen.

\begin{tabular}{|c|c|c|c|c|c|c|}
\hline & & $3 \mathrm{~m}$ & $6 \mathrm{~m}$ & $12 \mathrm{~m}$ & $24 \mathrm{~m}$ & $x^{2}(1)$ \\
\hline $\begin{array}{l}\text { Taux de nouaison(\%) } \\
\text { Fruit set rates (\%) }\end{array}$ & $\begin{array}{c}(a) \\
\text { Plein champ } \\
\text { In the open } \\
(b) \\
\text { Sous-cages } \\
\text { In cages } \\
(b) /(a) \\
\text { en } \%\end{array}$ & $\begin{array}{c}72( \pm 4) \\
19( \pm 4) \\
26\end{array}$ & $\begin{array}{c}55( \pm 5) \\
16( \pm 4) \\
29\end{array}$ & $\begin{array}{c}51( \pm 4) \\
12( \pm 2) \\
24\end{array}$ & $\begin{array}{c}49\left(\begin{array}{ll} \pm & 4\end{array}\right) \\
8\left(\begin{array}{ll} \pm & 1\end{array}\right) \\
16\end{array}$ & NS \\
\hline $\begin{array}{l}\text { Nombre de graines } \\
\text { par silique } \\
\text { Number of seeds per } \\
\text { siliqua }\end{array}$ & $\begin{array}{c}(a) \\
\text { Plein champ } \\
\text { In the open } \\
(b) \\
\text { Sous-cages } \\
\text { In cages } \\
(b) /(a) \\
\text { en } \%\end{array}$ & $\begin{array}{l}9( \pm 1) \\
2\left(\begin{array}{l} \pm \\
\pm\end{array}\right) \\
22\end{array}$ & $\begin{array}{c}8( \pm 1) \\
2( \pm 0,3) \\
25\end{array}$ & $\begin{array}{c}9\left(\begin{array}{l} \pm \\
)\end{array}\right. \\
1( \pm 0,1) \\
11\end{array}$ & $\begin{array}{c}10\left(\begin{array}{ll} \pm & 2\end{array}\right) \\
1\left(\begin{array}{cc} \pm & 0,1\end{array}\right) \\
10\end{array}$ & NS \\
\hline $\begin{array}{l}\text { Poids de } 1000 \text { grains } \\
\text { (en cg) } \\
\text { Weight of } 1000 \text { grains } \\
\text { (in cg) }\end{array}$ & $\begin{array}{c}(a) \\
\text { Plein champ } \\
\text { In the open } \\
(b) \\
\text { Sous-cages } \\
\text { In cages } \\
(b) /(a) \\
\text { en } \%\end{array}$ & $\begin{array}{c}430( \pm 20) \\
670( \pm 22) \\
156\end{array}$ & $\begin{array}{c}460( \pm 34) \\
660( \pm 12) \\
143\end{array}$ & $\begin{array}{c}380( \pm 4) \\
690( \pm 22) \\
182\end{array}$ & $\begin{array}{c}370( \pm 5) \\
700( \pm 31) \\
189\end{array}$ & NS \\
\hline $\begin{array}{l}\text { Production de graines } \\
\text { pour } 1000 \text { fleurs } \\
\text { Seed production of } \\
1000 \text { flowers }\end{array}$ & $\begin{array}{c}(a) \\
\text { Plein champ } \\
\text { In the open } \\
(b) \\
\text { Sous-cages } \\
\text { In cages } \\
(b) /(a) \\
\text { en } \%\end{array}$ & $\begin{array}{c}289( \pm 16) \\
34( \pm 18) \\
12\end{array}$ & $\begin{array}{c}204( \pm 19) \\
18( \pm 11) \\
9\end{array}$ & $\begin{array}{c}190( \pm 22) \\
13( \pm 7) \\
7\end{array}$ & $\begin{array}{c}195( \pm 53) \\
7( \pm \quad 0,4) \\
4\end{array}$ & NS \\
\hline $\begin{array}{l}\text { Rendement pour } 9 \mathrm{~m}^{2} \\
\text { (en } \mathrm{g} \text { ) } \\
\text { Yields for } 9 \mathrm{~m}^{2} \text { (in } \mathrm{g} \text { ) }\end{array}$ & $\begin{array}{c}(a) \\
\text { Plein champ } \\
\text { In the open } \\
(b) \\
\text { Sous-cages } \\
\text { In cages } \\
(b) /(a) \\
\text { en } \%\end{array}$ & $\begin{array}{c}1712( \pm 48) \\
146( \pm 99) \\
9\end{array}$ & $\begin{array}{c}1700( \pm 65) \\
151( \pm 57) \\
9\end{array}$ & $\begin{array}{c}41( \pm 9) \\
3\end{array}$ & $\begin{array}{c}52( \pm 16) \\
4\end{array}$ & * \\
\hline
\end{tabular}

Même légende que tableau 1 .

(1) Hypothèse : en fonction des différentes distances les résultats obtenus sous cages et de plein champ ne sont pas différents.

Same legend as Tabl. 1.

(1) Hypothesis: the pollination obtained in cages and in the open are not different over the various distances. 
On remarque aussi (Tabl. 1) que les toiles des cages utilisées ont laissé passer une bonne partie du pollen transporté par le vent par rapport aux récoltes de plein champ situées à la même distance ( 19 à $60 \%$, essai I, et 56 à $71 \%$, essai II) et que les captures sous cages peuvent être proportionnelles à celles de l'extérieur ( $\chi^{2}$ non significatif). Les pollens capturés ont été le plus souvent des pollens isolés, mais on a aussi noté fréquemment la présence de pollens groupés et parfois même en paquets.

\section{Incidence du pollen transporté par le vent sur les composantes du rendement}

Les tableaux 2 et 3 montrent que la pollinisation par le vent des plantes mâlestériles des cages a eu une très faible incidence sur les composantes du rendement par rapport à la pollinisation combinée vent + insectes de celles de l'extérieur : taux de nouaison plus faibles ( $4 \%$ à $29 \%$ ); siliques renfermant moins de graines ( $8 \%$ à $25 \%$ ); poids de 1000 grains plus élevés (108 à $189 \%$ ); productions grainières de 1000 fleurs et rendements plus faibles ( $1 \%$ à $12 \%$ ).

Il est intéressant de noter cependant la possibilité d'obtenir, par action du vent seul, des nouaisons sous cages, même si elles sont très faibles $(8 \% \pm 1$ et $1 \% \pm 0,2)$ à $24 \mathrm{~m}$ et à $32 \mathrm{~m}$ (Tabl. 2-3). Il est aussi intéressant de noter l'existence d'une certaine proportionnalité des gradients de pollinisation obtenus avec les composantes du rendement de l'intérieur des cages par rapport à celles de l'extérieur, sauf dans le cas de l'essai II où les $x^{2}$ des taux de nouaison et de la production de graines ont été très significatifs.

Notons enfin que la quantité de grains de pollen recueillie sous cages a été fortement correlée positivement avec le taux de nouaison $\left(r=0,84^{* * *}\right)$, le nombre de graines par silique $\left(r=0,84^{* * *}\right)$ et le rendement $\left(r=0,97^{* * *}\right)$. La corrélation a été au contraire négative avec le poids de 1000 grains $\left(r=-0,66^{*}\right)$ (phénomènes de compensation).

\section{Variations des récoltes de pollen en fonction de la direction $d u$ vent}

Les figures 2 et 3 font apparaitre des variations importantes entre captures de pollen effectuées de part et d'autre de la bande mâle-fertile émettrice de pollen des deux essais. Ces variations sont à rattacher, comme on pouvait s'y attendre, à l'influence de la force et de la direction du vent dominant. En effet, dans les deux cas, c'est le vent du $\mathrm{N}$ qui a dominé et ce sont les récoltes faites au $\mathrm{S}$ des bandes mâle-fertiles qui ont été les plus élevées dans un cas (Fig. 1) et à l'WSW dans l'autre cas (Fig. 2).

Les vitesses de vent enregistrées pendant toute la période des récoltes ont été comprises entre 3600 et $8600 \mathrm{~m} / \mathrm{h}$ en 1978 et entre 4300 et $12200 \mathrm{~m} / \mathrm{h}$ en 1979 .

\section{DISCUSSION}

Les résultats obtenus au cours de ces deux années d'observation permettent de confirmer ceux de Proctor et YEo (1973) sur différentes espèces à pollen anémophile; 
TABL. 3. - Comparaison de la pollinisation des colzas mâle-stériles plein champ et sous cages de l'essai II

à différentes distances des plantes mâle-fertiles émettrices de pollen.

TABL. 3. - Comparison of the pollination of male sterile rapeseeds in the open and in cages in test $I$

at various distances from the male fertile plants producing pollen.

\begin{tabular}{|c|c|c|c|c|c|c|}
\hline & & $1 \mathrm{~m}$ & $6 \mathrm{~m}$ & $15 \mathrm{~m}$ & $32 \mathrm{~m}$ & $x^{2}(1)$ \\
\hline $\begin{array}{l}\text { Taux de nouaison (\%) } \\
\text { Fruit set rates (\%) }\end{array}$ & $\begin{array}{c}(a) \\
\text { Plein champ } \\
\text { In the open } \\
\quad(b) \\
\text { Sous-cages } \\
\text { In cages } \\
\quad(b) /(a) \\
\text { en } \%\end{array}$ & $\begin{array}{c}56\left(\begin{array}{ll} \pm & 5\end{array}\right) \\
15\left(\begin{array}{l} \pm \\
1\end{array}\right) \\
27\end{array}$ & $\begin{array}{c}26( \pm 7) \\
6( \pm 1) \\
23\end{array}$ & $\begin{array}{c}37( \pm 5) \\
2( \pm 0,3) \\
5\end{array}$ & $\begin{array}{c}26( \pm 5) \\
1( \pm 0,2) \\
4\end{array}$ & $* * *$ \\
\hline $\begin{array}{l}\text { Nombre de graines par } \\
\text { silique } \\
\text { Number of seeds per siliqua }\end{array}$ & $\begin{array}{c}(a) \\
\text { Plein champ } \\
\text { In the open } \\
(b) \\
\text { Sous-cages } \\
\text { In cages } \\
(b) /(a) \\
\text { en } \% \\
\end{array}$ & $\begin{array}{c}12\left(\begin{array}{ll} \pm & 0,2\end{array}\right) \\
2\left(\begin{array}{ll} \pm & 0,3\end{array}\right) \\
17\end{array}$ & $\begin{array}{c}10( \pm 0,2) \\
1( \pm 0,1) \\
10\end{array}$ & $\begin{array}{c}12( \pm 2) \\
1( \pm 0,1) \\
8\end{array}$ & $\begin{array}{c}11( \pm 0,2) \\
1( \pm 0,1) \\
9\end{array}$ & NS \\
\hline $\begin{array}{l}\text { Poids de } 1000 \text { grains (en } \\
\text { cg) } \\
\text { Weight of } 1000 \text { grains (in cg) }\end{array}$ & $\begin{array}{c}(a) \\
\text { Plein champ } \\
\text { In the open } \\
(b) \\
\text { Sous-cages } \\
\text { In cages } \\
(b) /(a) \\
\text { en } \% \\
\end{array}$ & $\begin{array}{c}490( \pm 30) \\
810( \pm 40) \\
165\end{array}$ & $\begin{array}{c}580( \pm 10) \\
630( \pm 24) \\
109\end{array}$ & $\begin{array}{c}540( \pm 10) \\
650( \pm 42) \\
120\end{array}$ & $\left\{\begin{array}{c}620( \pm 25) \\
670( \pm 43) \\
108\end{array}\right.$ & NS \\
\hline $\begin{array}{l}\text { Production de graines pour } \\
1000 \text { fleurs } \\
\text { Seed production of } 1000 \\
\text { flowers }\end{array}$ & $\begin{array}{c}(a) \\
\text { Plein champ } \\
\text { In the open } \\
(b) \\
\text { Sous-cages } \\
\text { In cages } \\
(b) /(a) \\
\text { en } \% \\
\text { In cages }\end{array}$ & $319( \pm 6)$ & $151( \pm 33)$ & $242( \pm 41)$ & $\begin{array}{c}1\left(\begin{array}{cc} \pm & 0,2) \\
1\end{array}\right.\end{array}$ & $* * *$ \\
\hline
\end{tabular}

Même légende que tableau 1.

(1) Hypothèse : en fonction des différentes distances les résultats obtenus sous cages et de plein champ ne sont pas différents.

Same legend as Tabl. 1.

(1) Hypothesis: the pollination obtained in cages and in the open are not different over the various distances. 


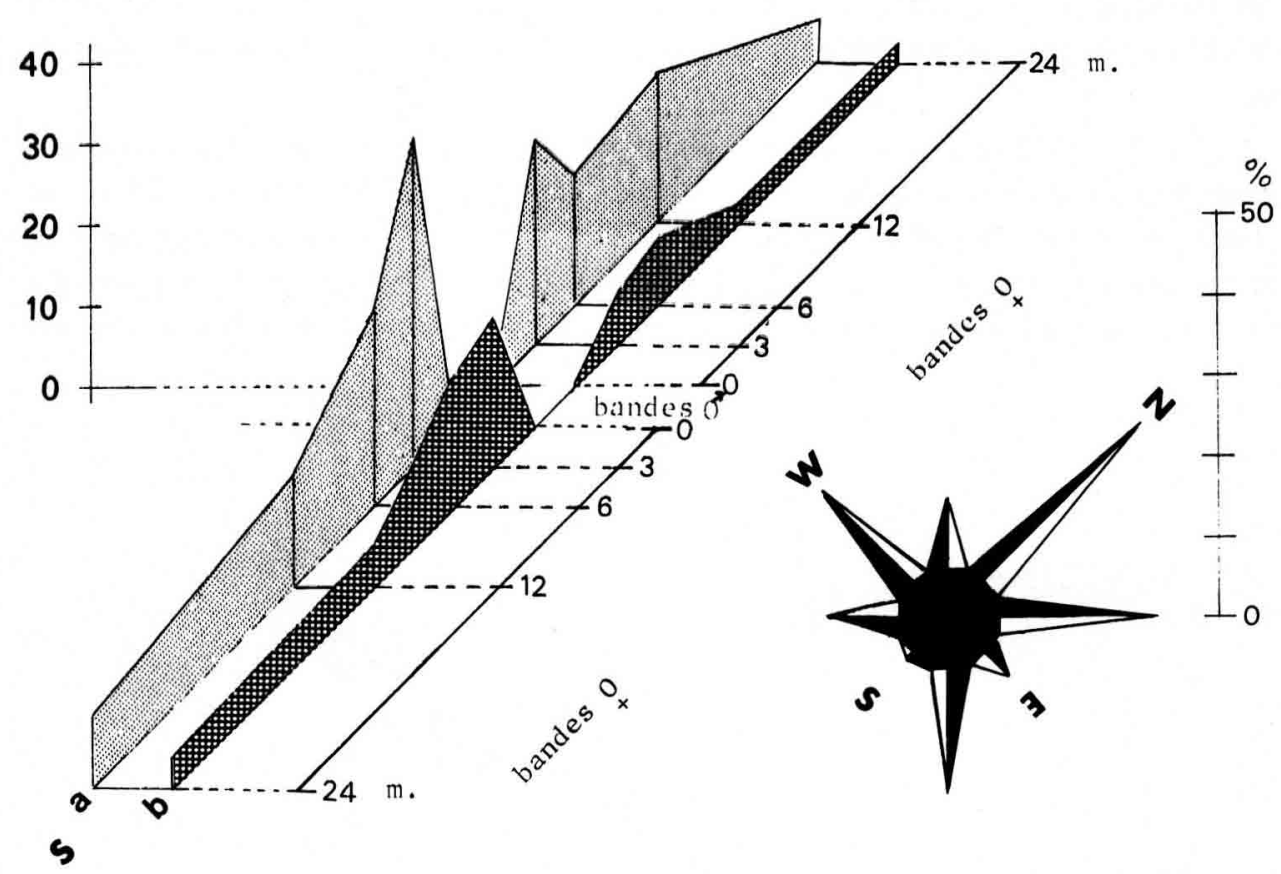

FIG. 2. - Distribution des captures des grains de pollen dans les colzas mâle-stériles et à différentes distances des bandes mâle-fertiles. Essai I.

En abscisse : les distances en mètre.

En ordonnées : nombre de grains de pollen capturés par 24 heures et par centimètre carré.

a) Captures de plein champ.

b) Captures de l'intérieur des cages.

FIG. 2. - Dispersal of pollen grains in male sterile rapeseeds $x$-axis : distances in $\mathrm{m}$. at various distances from the male fertile strip. Test $I$.

$y$-axis : number of pollen grains per $\mathrm{cm}^{2}$ caught per 24 hours.

a) Catchings in the open.

b) Catchings inside the cages.

BATEMAN (1947 $a$ et $b$ ) sur chou; Olsson (1955) sur colza en ce qui concerne la répartition des captures au champ des grains de pollen transportés par le vent. Les plus grósses captures ont toujours lieu près de la source émettrice de pollen, elles diminuent rapidement ensuite et elles peuvent se maintenir dans de faibles proportions sur des distances plus ou moins longues. La dispersion du pollen de colza par le vent, comme l'a d'ailleurs obtenue Olsson (1955) a tendance à se faire selon une distribution sensiblement plus aplatie que celle donnée par Proctor et Yeo qu'ils assimilent à une distribution du type leptocurtique. Nous pensons que cette différence peut s'expliquer par le fait que Proctor et Yeo ont travaillé uniquement sur du pollen typiquement anémophile, 
donc plus léger, et d'une texture différente du pollen de colza. La distribution d'un pollen anémophile transporté par le vent sur de plus longues distances $(500 \mathrm{~m})$ peut dans ce cas ne pas être comparable et prendre une allure différente de celle du pollen de colza.

Ainsi, le vent peut jouer néanmoins un certain rôle dans la pollinisation du colza, comme cela a d'ailleurs été signalé par Nicolaisen (1943) et OLSSON (1955). Ce sont surtout les grains de pollen dispersés par le vent près de sa source émettrice qui sont certes plus importants pour la pollinisation, en raison de leur plus grand nombre, que ceux transportés à longue distance. Mais dans tous les cas, le vent ne suffit pas à lui seul

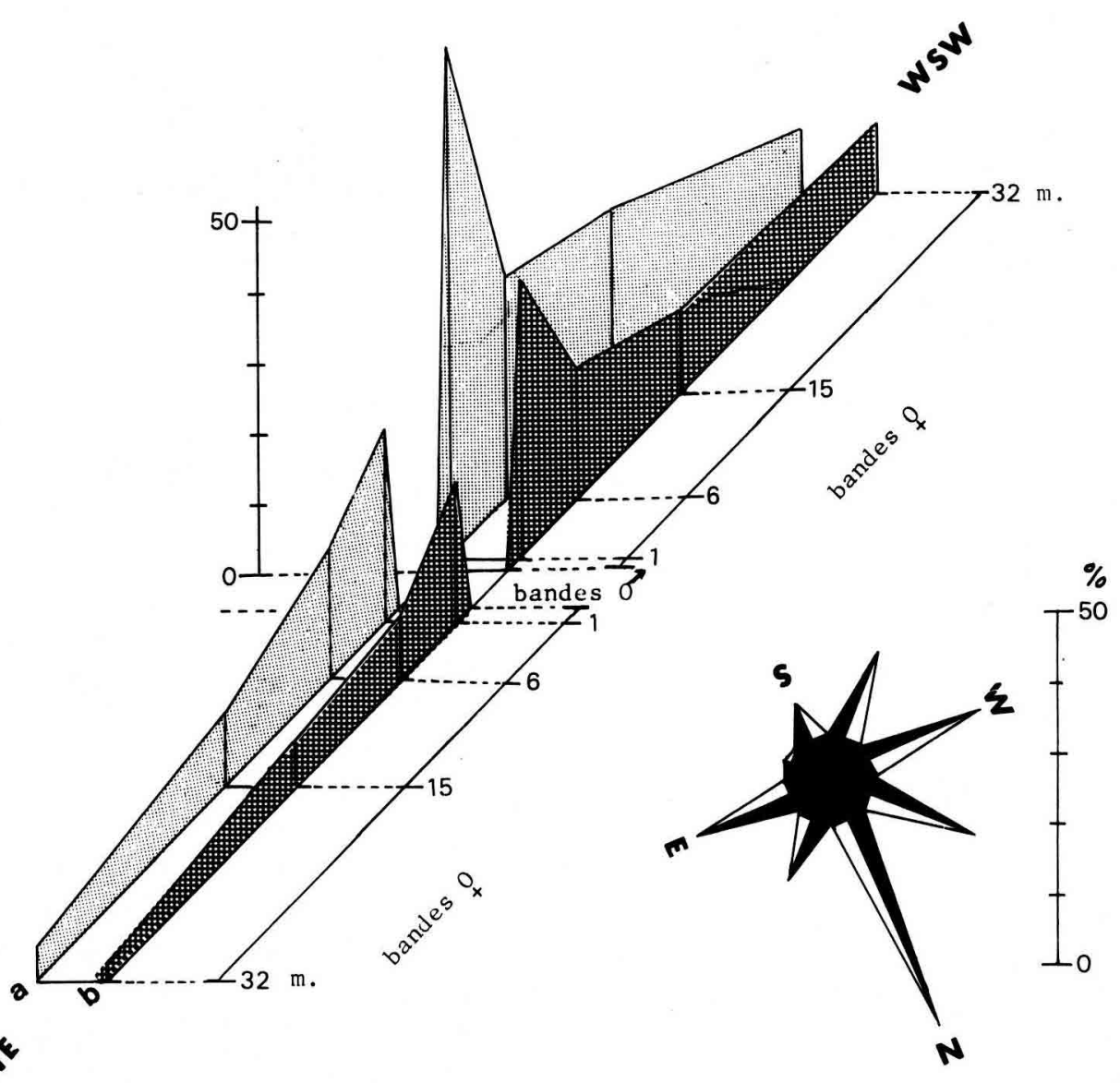

Fıg. 3. - Distribution des captures des grains de pollen dans les colzas mâle-stériles $\grave{a}$ différentes distances des bandes mâle-fertiles. Essai $I I$. Même légende que la figure 2.

FIG. 3. - Dispersal of pollen grains in male sterile rapeseeds at various distances from the male fertile strip. Test II.

Same legend as Fig. 2. 
pour assurer la pollinisation et la production grainière satisfaisante du colza mâlestérile; s'il favorise l'autopollinisation chez les plantes mâle-fertiles par secouement des plantes (WILliams, 1978), il semble jouer aussi un rôle dans la pollinisation croisée du colza. L'incidence du vent sur la pollinisation du colza mâle-stérile s'observe assez nettement sur les taux de nouaison. En effet, les nouaisons des plantes mâle-stériles des cages dues à l'action du vent seul sont assez élevées près de la bande mâle-fertile (on a trouvé des taux de nouaison à peu près semblables lorsqu'on étudiait l'action du vent sur la pollinisation des mâle-stériles en présence de mâle-fertiles dans les mêmes cages (Mesquida et Renard, 1981). Elles diminuent ensuite au fur et à mesure que l'on s'éloigne de la source émettrice de pollen selon un gradient de même pente mais avec des taux plus bas que dans le cas de l'action combinée du vent et des insectes.

JENKINSON et GLYNNE-JONEs (1953) ont d'ailleurs observé sur chou et colza que le pollen transporté par le vent se présente sous forme d'un dépôt de quelques grains de pollen dispersés irrégulièrement sur les stigmates alors que ceux visités par les abeilles ont de nombreux grains de pollen déposés sur toute la surface. Cette hypothèse pourrait fort bien expliquer la faible efficacité du pollen transporté par le vent sur nos plantes mâle-stériles.

Ces observations ont permis finalement de constater :

- que les grains de pollen de colza peuvent être transportés par le vent jusqu'à $32 \mathrm{~m}$ de la source émettrice de pollen;

- que les quantités de grains de pollen recueillies sont plus importantes (75\% environ de la récolte totale) à moins de $6 \mathrm{~m}$, et qu'elles diminuent rapidement ensuite pour rester à un niveau assez bas;

- que les cages utilisées laissent passer environ 19 et $71 \%$ des grains de pollen véhiculés par le vent;

- que les grains de pollen transportés sur de grandes distances n'ont pas une grande incidence sur le rendement en graines sous cages, mais dans les conditions de nos expérimentations, 23 à $29 \%$ de la production de siliques et 3 à $12 \%$ de la production grainière semblent imputables à l'action du vent seul à moins de $6 \mathrm{~m} ; 4$ à $16 \%$ de la production de siliques et 1 à $4 \%$ de production grainière seulement sont dus au vent à plus de $24 \mathrm{~m}$.

\section{REMERCIEMENTS}

Nous tenons à remercier tout particulièrement l'I.T.A.P.I. (1) et P.R.O.M.O.S.O.L. (2) pour l'aide que ces deux organismes nous ont apportée.

Reçu pour publication en mai 1982.

Eingegangen im Mai 1982.

(1) I.T.A.P.I. : Institut technique de l'apiculture, station de recherches sur l'abeille et les insectes sociaux, La Guyonnerie, 91440 Bures sur Yvette.

(2) P.R.O.M.O.S.O.L. : Association pour la promotion de la sélection des semences oléagineuses, 12, avenue George-V, 75008 Paris. 


\section{ZUSAMMENFASSUNG}

\section{UNTERSUCHUNG DER POLLENVERFRACHTUNG DURCH DEN WIND UND DIE BEDEUTUNG} DER WINDBESTÄUBUNG BEI RAPS (BRASSICA NAPUS L., VAR, OLEIFERA METZGER).

In den Jahren 1978 und 1979 haben wir in Rennes (Ille-et-Vilaine) zwei Versuche (Versuch I und II) über die Verteilung des Rapspollens durch den Wind durchgeführt mit dem Ziel, bei einer Versuchsanordnung im Rahmen unserer Experimente zur Erzeugung von F 1-Hybridsaaten (alternierende Aussaat von fertilem und sterilem Raps) den Anteil der Windbestäubung zu bestimmen.

\section{Material und Methoden}

Es wurde ein Pollenspender, ein männlich-fertiler Raps (Varietät "Primor »), und eine männlichsterile Sorte (aus zytoplasmatischer Ursache) benutzt, um den Wirkungsgrad des durch den Wind transportierten Pollens zu bestimmen.

Jeder Versuch wurde in einer Parzelle $60 \times 30 \mathrm{~m}$ durchgeführt, die in Richtung ihrer Breite zwei Streifen (Totalbreite $6 \mathrm{~m}$ ) von männlich-fertilem Raps enthielt, an jeder Seite umgeben von zehn Streifen (Gesamtbreite $35 \mathrm{~m}$ ) mit männlich-sterilem Raps. Jeder Streifen bestand aus 8 Reihen mit $40 \mathrm{~cm}$ Zwischenraum und mit $70 \mathrm{~cm}$ Abstand zwischen den Streifen.

Die männlich-sterilen Streifen befanden sich bei Versuch I nördlich und südlich der männlich-fertilen Streifen und bei Versuch II ONO und WSW von ihnen.

Die Pollen-Fangeinrichtungen (Kollektoren, Fig. 1) wurden in den männlich-sterilen Streifen aufgestelit, und zwar bei Versuch I in einer Entfernung von $3 \mathrm{~m}, 6 \mathrm{~m}, 12 \mathrm{~m}$ und $24 \mathrm{~m}$, und bei Versuch II in einer Entfernung von $1 \mathrm{~m}, 6 \mathrm{~m}, 15 \mathrm{~m}$ und $32 \mathrm{~m}$. An jeder Fangstelle wurden zwei Pollenkollektoren aufgestellt, einer ausserhalb und einer innerhalb eines Zeltes $(3 \times 3 \times 2 \mathrm{~m})$ aus Plastikgewebe von $2 \mathrm{~mm}$ Maschenweite zur Verhinderung des Insektenbefluges. Jeder Käfig bedeckte $9 \mathrm{~m}^{2}$ von männlich-sterilem Raps, dessen Bestäubung ausschliesslich vom Wind abhing. Im Freiland erfolgte die Bestäubung der männlich-sterilen Rapsblüten sowohl durch Insekten wie durch den Wind.

Die Pollenkörner wurden mittels aufrecht stehender Glasplatten $(76 \times 26 \mathrm{~mm})$, überzogen mit einer dünnen Schicht Glyzerin, aufgefangen.

Die Beobachtungsdaten geben die Fangresultate von 24 Stunden (Zahl der Körner pro $\mathrm{cm}^{2}$ ) während der Blütezeit des Raps wieder, abgesehen von Regentagen. Die Bestäubung der männlich-sterilen Pflanzen im Käfig und im Freiland wurde nach der Rate des Fruchtansatzes, der Zahl der Samenkörner pro Schote, dem Gewicht von 1000 Körnern, der Samenproduktion pro 1000 Blüten und dem Ertrag der ganzen Pflanzen bestimmt.

\section{Resultate}

1. Wir haben Pollen bis zu $32 \mathrm{~m}$ Entfernung von dem pollenspendenden männlich-sterilen Streifen gefunden (Tabl. 1). Der Grossteil ( $75 \%$ des Gesamtpollens) wurde jedoch innerhalb von $6 \mathrm{~m}$ aufgefangen. Immerhin wurden 7-10\% des Gesamtpollens zwischen 24-32 m Entfernung geerntet. Das benutzte Gewebe der Käfige liess zwischen 19 und $71 \%$ des vom Wind transportierten Pollens passieren.

2. Im Vergleich zu der kombinierten Bestäubung Wind + Insekten im Freiland, war der Windeffekt innerhalb der Käfige auf die Ertragsfaktoren sehr gering. Der Fruchtansatz war niedriger (4-29\%), die Schoten enthielten sehr wenig Samenkörner (8-25\%), die jedoch grösser waren (108-189\%) und die Samenproduktion pro 1000 Blüten war gleichermassen geringer wie die Erträge (1-12\%; Tabl. 2 und 3). 
Es wurden signifikante positive Korrelationen zwischen dem unter dem Netz aufgefangenen Pollen und dem Grad des Fruchtansatzes $\left(r=0,84^{* * *}\right)$, der Anzahl der Samenkörner pro Schote $\left(r=0,84^{* * *}\right)$ und dem Ertrag $\left(r=0,97^{* * 7}\right)$ gefunden. Die Korrelation war negativ für das 1000 Korngewicht $\left(r=0,66^{* *}\right.$; Kompensationsphänomen).

3. Die Abbildungen 2 und 3 zeigen einen starken Einfluss der Hauptwindrichtung. Bei einer Hauptwindrichtung von N waren die Fänge in einem Fall im S am höchsten (Abb. 2), im anderen in WSW (Abb. 3). Die Windgeschwindigkeit lag zwischen 3600 und $8600 \mathrm{~m}$ im Jahre 1978 und zwischen 4300 und $12200 \mathrm{~m} / \mathrm{h}$ im Jahre 1979.

4. Wind kann bei der Bestäubung der Rapsblüten eine gewisse Rolle spielen. Die Pollenübertragung durch den Wind über grössere Entfernungen hatte im Käfig keinen grossen Einfluss auf den Fruchtansatz, aber innerhalb von $6 \mathrm{~m}$ scheinen 23-29\% der Samenproduktion der alleinigen Wirkung des Windes zuzuschreiben zu sein. In einer Entfernung von mehr als $24 \mathrm{~m}$ bewirkt der Wind nur $4-16 \%$ der Schotenerzeugung und 1-4\% der Erzeugung von Samenkörnern.

\title{
SUMMARY
}

\author{
STUDY OF THE POLLEN DISPERSAL BY WIND AND OF THE IMPORTANCE \\ OF WIND-POLLINATION IN RAPESEED (BRASSICA NAPUS VAR. OLEIFERA METZGER).
}

In 1978 and 1979 we made 2 tests (tests I and II) in Rennes (Brittany) on rapeseed pollen dispersal by wind to determine the proportion of wind-pollination in the devices that alternate fertile ans sterile rapeseeds in our tests of $F 1$ hybrid seed production.

\section{Materials and methods}

We used a male fertile rapeseed ("Primor " cultivar) as pollen producer and a cytoplasmic male sterile rapeseed as pollen receiver to test the efficiency of pollen transferred by wind.

Each test was carried out in a $60 \times 30 \mathrm{~m}$ plot that included in its width two strips (total width : $6 \mathrm{~m}$ ) of male fertile rapeseed bounded on each side by ten strips (total width: $35 \mathrm{~m}$ ) of male sterile rapeseed. Each strip was composed of 8 rows with a $0,40 \mathrm{~m}$ between plants and $0,70 \mathrm{~m}$ between rows.

Male sterile strips were situated on the north and the south of male fertile strips in test $I$ and ENE and WSW in test II.

Pollen collectors (Fig. 1) were put in male sterile strips at a distance of $3 \mathrm{~m}, 6 \mathrm{~m}, 12 \mathrm{~m}$ and $24 \mathrm{~m}$ in test $I$ and $1 \mathrm{~m}, 6 \mathrm{~m}, 15 \mathrm{~m}$ and $32 \mathrm{~m}$ in test II. At each catching distance one pollen collector was put in the open and a second one in $3 \times 3 \times 2 \mathrm{~m}$ cages covered with a $2 \mathrm{~mm}$ mesh plastic net that prevented access of pollinating insects. Wind-pollination was evaluated in cages by covering $9 \mathrm{~m}^{2}$ of male sterile rapeseed. In the case of open pollination of male sterile rapeseed, pollination was performed both by insects and wind.

Pollens were caught on $76 \times 26 \mathrm{~mm}$ glass slides covered with a thin layer of glycerin and kept upright on the collector.

Observations relating to 24-hour catchings (number of grains per $\mathrm{m}^{2}$ ) were made during the flowering time of rapeseed except in wet weather. Pollination of male sterile plants in cages and in the open was confirmed by fruit set rates, number of seeds per siliqua, weight of 1000 grains, seed production of 1000 flowers and yields of whole plants. 


\section{Results and conclusion}

1. Pollen was captured up to $32 \mathrm{~m}$ from the male fertile strip producing pollen (Tabl. 1). The highest catchings took place within $6 \mathrm{~m}$ (75\% of the total pollen). Yet 7-10\% of the total pollen was caught between 24 and $32 \mathrm{~m}$. The cage nets allowed between $19 \%$ to $71 \%$ pollen transfer by wind.

2. With respect to the wind and insects combined pollination of plants in the open, the wind effect in cages had a very low influence on the yield. Fruit set rates were lower (8-25\%), siliquae contained very few seeds (8-25\%), the weights were larger (108-189\%) and seed production of 1000 flowers as well as yields were lower (1-12\%) (Tabl. 2 and 3). Significant positive correlations were found between pollen captured in cages and fruit set rates $\left(r=0,84^{* * *}\right)$ number of seeds per siliqua $\left(r=0,84^{* * *}\right)$, and yields $\left(r=0,97^{* * *}\right)$. There was a negative correlation between the weight of 1000 grains $(r=0,6699)$ compensating phenoma) and the pollen captured.

3. Figures 2 and 3 show a strong influence of the main wind. In main north wind, south catchings were the most numerous in one case (Fig. 2) and the WSW ones in the other cases (Fig. 3). Wind speeds were 3600 and $8000 \mathrm{~m} / \mathrm{h}$ in 1978 and 4300 and $12200 \mathrm{~m} / \mathrm{h}$ in 1979.

4. Wind can play a role in rapeseed pollination. Pollens transferred by wind over long distance did not have a great influence on the fruit set in cages but $23-29 \%$ of the seed production seemed to be attributable to the sole action of wind within $6 \mathrm{~m}$; beyond $24 \mathrm{~m}$ the percentages decreased respectively to 4-16\% and $1-4 \%$.

\section{RÉFÉRENCES BIBLIOGRAPHIQUES}

Bateman A. J., 1947 (a). - Contamination of seed crops. II. Wind pollination. Heredity, 1, 235-246.

BATEMAN A. J., 1947 (b). - Contamination in seed crops. III. Relation with isolation distance. Heredity, 1, 303-336.

Dagnele P., 1969. - Théorie et méthodes statistiques. Applications agronomiques. Duculot S.A., Gembloux.

GrIFFITHS D. J., 1950. - The liability of seed-crops of perrennial Rye Grass (Lolium perenne) to contamination by wind-borne pollen. J. Agric. Sci., Camb., 40, 19-38.

HYDE H. A., 1959. - Volumetric counts of pollen grains at Cardiff 1954-1957. The journal of allergy, 30, 219-234.

Hyde H. A. et Williams D. A., 1945. - Studies in atmospheric pollen. II. Diurnal variation in the incidence of grass pollen. Newphytologist, 44, 83-94.

Jenkinson J. G. et GLYNNE-Jones G. D., 1953. - Observations on the pollination of oil rape and Broccoli (Brassica napus and B. oleracea). Bee World, 34, 173-174.

LouveauX J., 1958. - Recherches sur la récolte du pollen par les abeilles (Apis mellifica L.). Thèse Fac. Sci. Univ. Paris, 206 p.

Maurizıo A. et Louveaux J., 1965. - Pollens de plantes mellifères d'Europe. Union des Group. Apic. F. Paris.

Mesquida J. et ReNARD M., 1978. - The entomophilous pollination of mâle-sterile strains of winter Rapeseed (Brassica napus L. var. oleifera Metzger) and a preliminary study of alternating devices. Proceedings of the fourth Intern. Symp. on pollination. Maryland, 49-57.

Mesquida J. et ReNARD M., 1981. - Pollinisation du colza d'hiver mâle-fertile et mâle-stérile (Brassica napus L. var. oleifera Metzger) par l'abeille domestique (Apis m. mellifica L.). Effets sur la phénologie et le rendement. Apidologie, 12, 345-362.

Nicolaisen W., 1943. - Züchtung von Raps. Z. Pflanzenzüchtg., 25, 362-379. 
Ousson G., 1955. - Vindpollinering hos korsblomstriga oljeväxter (in Swedish English Summary). Sver. Utsadesfoi., 65, 418-422.

Olsson G. et Persson B., 1958. - Inkorsningsgrad och självsterilitet hos raps (in Swedish, English Summary). Sver. Utsadesfoi., 68, 74-78.

Proctor M. et Yeo P., 1973. - The pollination of flower Collins, London, $407 \mathrm{p}$.

Wang C. W., Perry T. O. et Johnson A. G., 1960. - Pollen dispersion of Slash Pine (Pinus elliottii Engelm) with special reference to seed orchard management. Silvae Genet., 9, 78-86.

WiLliams I. H., 1978. - The pollination requirements of swede rape (Brassica napus L.) and of turnip rape (Brassicae campestris L.). J. Agric. Sci., Camb., 91, 343-348.

WRIGHT J. W., 1953. - Pollen-dispersion studies : some practical applications. J. Forestry, 51, 114-118. 